number variation to a polycomb/trithorax epigenetic switch in FSHD muscular dystrophy. Cell. 2012;149(4):819-831.

17. Jeck WR, Siebold AP, Sharpless NE. Review: a metaanalysis of GWAS and age-associated diseases. Aging Cell. 2012;11(5):727-731.

18. Harismendy O, et al. 9p21 DNA variants associated with coronary artery disease impair interferon- $\gamma$ sig- nalling response. Nature. 2011;470(7333):264-268.

19. Liu Y, et al. INK4/ARF transcript expression is associated with chromosome $9 \mathrm{p} 21$ variants linked to atherosclerosis. PLoS One. 2009;4(4):e5027.

20. Burd CE, Jeck WR, Liu Y, Sanoff HK, Wang Z, Sharpless NE. Expression of linear and novel circular forms of an INK4/ARF-associated non-coding RNA correlates with atherosclerosis risk. PLoS
Genet. 2010;6(12):e1001233.

21. Lachmeijer AM, et al. A genome-wide scan for preeclampsia in the Netherlands. Eur J Hum Genet. 2001;9(10):758-764.

22. Maass PG, et al. A cis-regulatory site downregulates PTHLH in translocation $\mathrm{t}(8 ; 12)(\mathrm{q} 13 ; \mathrm{p} 11.2)$ and leads to Brachydactyly Type E. Hum Mol Genet. 2010;19(5):848-860

\title{
Tipping the scales early: probing the long-term effects of obesity
}

\author{
Chen Liu and Joel K. Elmquist
}

\author{
Division of Hypothalamic Research, Departments of Internal Medicine and Pharmacology, \\ University of Texas Southwestern Medical Center at Dallas, Dallas, Texas, USA.
}

\begin{abstract}
Obesity has reached epidemic proportions in the United States, and obesityrelated illnesses have become a leading preventable cause of death. Childhood obesity is also growing in frequency, and the impact of a lifetime spent in the overweight state is only beginning to emerge in the literature. In this issue of the JCI, Bumaschny et al. used a genetic mouse model to investigate the self-perpetuating nature of obesity and shed some light on why it can become increasingly difficult to lose weight over time.
\end{abstract}

The global pandemic of obesity affects the health of more than 500 million people. Obesity poses a major risk for other comorbid diseases and has become a leading preventable cause of death in the United States. For morbidly obese patients, a modest $(5 \%-10 \%)$ reduction in body weight can bring significant health benefits such as improvements in blood pressure and glycemic control, which may ultimately contribute to decreased mortality (1). Nevertheless, despite lifestyle interventions and numerous efforts in developing effective anti-obesity therapies, sustained weight management remains a challenge for most obese patients.

Limited efficacy and weight rebound are two common problems, yet the mechanisms underlying treatment refractoriness remain to be identified. It is generally believed that chronic obesity may trigger maladaptive responses that help retain a state of sustained positive energy balance. However, given the complex genetic, environmental, and social factors involved in the etiology of obesity, it has been very difficult to evaluate the impact of chronic obesity itself on the effectiveness of anti-obesity therapies.

Conflict of interest: The authors have declared that no conflict of interest exists.

Citation for this article: J Clin Invest. 2012; 122(11):3840-3842. doi:10.1172/JCI66409.
In this issue of the JCI, Bumaschny and colleagues generated a novel mouse obesity model in which severe and early-onset obesity is induced by the loss of a single gene, proopiomelanocortin (Pomc), in a small population of neurons in the brain (2). Notably, the model allowed the authors to "treat" the obesity by restoring Pomc expression at different ages and directly test the efficacy of gene therapy after different durations of the obese state.

\section{Reversible genetic obesity}

The Pomc gene encodes a polypeptide that is most abundant in the anterior pituitary gland and in neurons in the arcuate nucleus of the hypothalamus (ARH). The 241-amino-acid polypeptide is the precursor of many physiologically important molecules, including the anorexigenic peptide $\alpha$-melanocyte-stimulating hormone ( $\alpha-\mathrm{MSH})$ (3). Mutations of the Pomc gene, in both rodents and humans, lead to severe early onset of obesity as well as adrenal insufficiency that is the result of deficiency in another Pomc product, adrenocorticotropic hormone (ACTH) (4-6).

A major strength of the work of Bumaschny et al. is the generation and characterization of several elegant genetic tools for in vivo obesity studies. First, in order to generate a Pomc-deficient obesity model without pituitary dysfunction, Bumas- chny et al. took advantage of their previous findings that Pomc expression in the pituitary gland and hypothalamus is governed by two independent upstream regulatory sequences (7) and engineered a new knockin mouse line in which Pomc transcription is selectively disrupted specifically in hypothalamic neurons (2). As expected, these mice developed early-onset obesity due to the lack of hypothalamic Pomc expression, whereas Pomc expression in pituitary cells remained undisturbed. An important feature of this model is that physiological levels of Pomc expression can be restored by tamoxifen-inducible Cre-mediated recombination (8), so that the hypothalamic Pomc deficiency can be reversed at different postnatal stages in a temporally controlled manner.

\section{Timing is everything}

Bumaschny and colleagues found that restoration of neuronal Pomc expression in lean juvenile mice completely prevented the subsequent development of obesity. However, the ability of hypothalamic Pomc reexpression to normalize body weight was progressively attenuated as the age of Pomc rescue was delayed and the obesity was allowed to continue. Given that the degree of Pomc restoration remained constant among all age groups, Bumaschny and colleagues suspected that the increased adiposity and the metabolic changes associated with obesity played a major role in the blunted phenotypic responses to Pomc rescue. In an innovative approach, the authors tested this hypothesis using a caloric restriction regimen to delay the onset of obesity in older Pomcdeficient mice. At P60, when obesity is evi- 


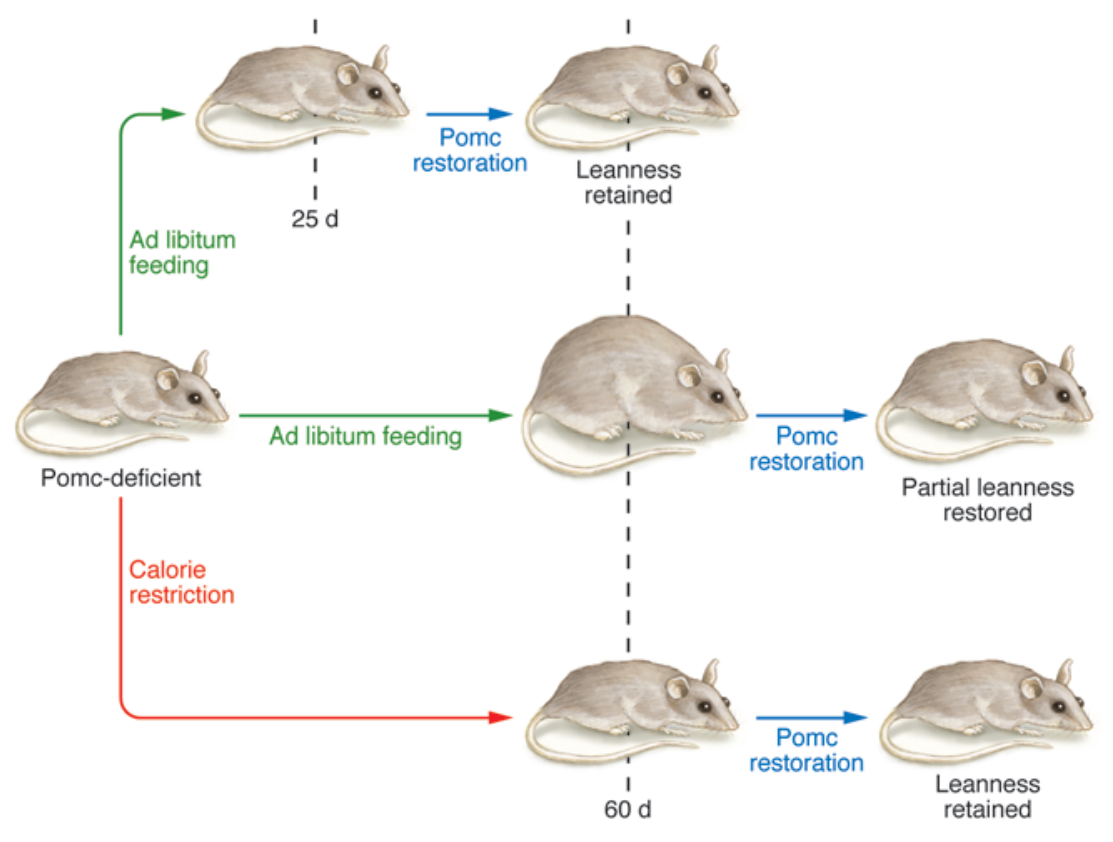

dent in Pomc-deficient mice fed ad libitum and Pomc reexpression can only partially alleviate the development of obesity, the calorie-restricted Pomc-deficient mice had body weight comparable to that of wildtype controls. Furthermore, Pomc restoration in calorie-restricted mice successfully prevented the subsequent development of obesity. These results therefore indicate that the preceding development of obesity and its cumulative effects, but not age, are responsible for the attenuated phenotypic responses to Pomc rescue in older Pomcdeficient mice (Figure 1).

A key point of the work of Bumaschny et al. is the potential importance of early intervention for the effective treatment of obesity and its associated comorbid conditions. This is particularly relevant in our society today. For example, more than one-third of children and adolescents are overweight or obese in the United States (9), and the rates are increasing. If left unmanaged, obesity in childhood is likely to persist into adulthood (10), and, as indicated by Bumaschny et al., these individuals may become resistant to later medical interventions. Indeed, the authors' finding that, despite the significant normalization of hyperphagia, the improvement in body weight progressively waned as the restoration of Pomc expression was delayed supports the notion that obesity is a self-perpetuating condition that needs early management and intervention. Finally, it has become increasingly clear that alterations in the metabolic state of moth- ers during critical periods of fetal development, both in utero and in the early postnatal period, can have long-lasting effects in the offspring $(11,12)$. Thus, early intervention to combat obesity may become even more of an issue as more and more children are born in the context of maternal obesity and diabetes.

\section{Hungry for more}

Despite these provocative and potentially important results, relatively little is known about how obesity induces maladaptive changes that permanently alter normal body weight homeostasis. It is conceivable that being chronically overweight may alter the structure and function of brain circuits that mediate the central control of energy homeostasis. This possibility for "plasticity" in the hypothalamus has been highlighted in recent studies $(13,14)$. Moreover, previous studies have implicated obesity-associated hyperleptinemia (15), chronic inflammation (16), ER stress (17), and changes in reactive oxygen species (18) in the alterations in hypothalamic neurons. Additionally, a recent study found reduced adult hypothalamic neurogenesis in mice with diet-induced obesity (DIO) and in leptindeficient $o b / o b$ mice, suggesting obesity also affects the dynamic turnover and remodeling of the hypothalamic neurons (19).

Bumaschny et al. made another important finding by showing that although Pomc restoration is effective in normalizing food intake, it does not correct the

\section{Figure 1}

Pomc deletion in the hypothalamus leads to obesity in ad libitum fed mice. Bumaschny and colleagues demonstrated that the restoration of hypothalamic Pomc expression could normalize body weight in young mice, but this is progressively attenuated as obesity was allowed to continue and the age of Pomc restoration was delayed. When Pomc-deficient mice were aged on a calorie-restricted diet, later Pomc reexpression prevented the subsequent development of obesity, suggesting that the preceding development of obesity, but not age itself, is responsible for the attenuated phenotypic responses to Pomc rescue in older Pomc-deficient mice. defects in energy expenditure after obesity is established. This finding raises the possibility that obesity may induce maladaptive and irreversible changes that selectively affect neural circuits that regulate energy expenditure and is consistent with previous findings that food intake and energy expenditure are regulated by distinct neural pathways downstream of hypothalamic Pomc neurons (20). Finally, the elegant mouse model Bumaschny et al. describe could be used to probe any number of other physiological processes that have been ascribed to Pomc neurons, including the regulation of glucose homeostasis, physical activity, and mood regulation (3).

\section{Conclusions}

In summary, with the increasing prevalence of obesity and the paucity of effective treatments, it is imperative to design new strategies to halt the obesity epidemic. By using a novel reversible monogenic obesity model, Bumaschny et al. explored the temporal window for effective treatment and provided new insights into the mechanisms underlying treatment refractoriness. These findings highlight the potential necessity of early intervention in the treatment of obesity and have important clinical implications for the management of childhood obesity. Moreover, these studies extend our current understanding of obesity progression and suggest more attention should be focused on reversing the obesity-associated maladaptive changes in the brain. 


\section{Acknowledgments}

C. Liu is supported by a postdoctoral fellowship from the Hilda and Preston Davis Foundation. J.K. Elmquist is supported by NIH grants RL1 DK081185, R37 DK053301, and P01 DK088761.

Address correspondence to: Joel K. Elmquist, Division of Hypothalamic Research, UT Southwestern Medical Center, 5323 Harry Hines Blvd., Dallas, Texas 75390, USA. Phone: 214.648.2911; Fax: 214.648.5612; E-mail: joel.elmquist@ utsouthwestern.edu.

1. Poobalan AS, Aucott LS, Smith WC, Avenell A, Jung R, Broom J. Long-term weight loss effects on all cause mortality in overweight/obese populations. Obes Rev. 2007;8(6):503-513.

2. Bumaschny VF, et al. Obesity-programmed mice are rescued by early genetic intervention. J Clin Invest. 2012;122(11):4203-4212.

3. Cone RD. Anatomy and regulation of the central melanocortin system. Nat Neurosci. 2005;8(5):571-578.

4. Yaswen L, Diehl N, Brennan MB, Hochgeschwender U. Obesity in the mouse model of proopiomelanocortin deficiency responds to peripheral melanocortin. Nat Med. 1999;5(9):1066-1070.
5. Krude H, Biebermann H, Luck W, Horn R, Brabant G, Gruters A. Severe early-onset obesity, adrenal insufficiency and red hair pigmentation caused by POMC mutations in humans. Nat Genet. 1998;19(2):155-157.

6. Challis BG, et al. Mice lacking pro-opiomelanocortin are sensitive to high-fat feeding but respond normally to the acute anorectic effects of peptide-YY(3-36). Proc Natl Acad Sci U S A. 2004;101(13):4695-4700.

7. de Souza FS, et al. Identification of neuronal enhancers of the proopiomelanocortin gene by transgenic mouse analysis and phylogenetic footprinting. Mol Cell Biol. 2005;25(8):3076-3086.

8. Feil R, Brocard J, Mascrez B, LeMeur M, Metzger $D$, Chambon P. Ligand-activated site-specific recombination in mice. Proc Natl Acad Sci U S A. 1996;93(20):10887-10890.

9. Ogden CL, Carroll MD, Curtin LR, Lamb MM, Flegal KM. Prevalence of high body mass index in US children and adolescents, 2007-2008. JAMA. 2010;303(3):242-249.

10. Freedman DS, Dietz WH, Srinivasan SR, Berenson GS. The relation of overweight to cardiovascular risk factors among children and adolescents: the Bogalusa Heart Study. Pediatrics. 1999; 103(6 pt 1):1175-1182.

11. Sullivan EL, et al. Chronic consumption of a highfat diet during pregnancy causes perturbations in the serotonergic system and increased anxiety-like behavior in nonhuman primate offspring. J Neurosci. 2010;30(10):3826-3830.
12. McMillen IC, Robinson JS. Developmental origins of the metabolic syndrome: prediction, plasticity, and programming. Physiol Rev. 2005;85(2):571-633.

13. Pinto $S$, et al. Rapid rewiring of arcuate nucleus feeding circuits by leptin. Science. 2004; 304(5667):110-115

14. Liu T, et al. Fasting activation of AgRP neurons requires NMDA receptors and involves spinogenesis and increased excitatory tone. Neuron. 2012;73(3):511-522.

15. Munzberg H, Flier JS, Bjorbaek C. Regionspecific leptin resistance within the hypothalamus of diet-induced obese mice. Endocrinology. 2004;145(11):4880-4889.

16. De Souza CT, et al. Consumption of a fat-rich diet activates a proinflammatory response and induces insulin resistance in the hypothalamus. Endocrinology. 2005;146(10):4192-4199.

17. Ozcan U, et al. Endoplasmic reticulum stress links obesity, insulin action, and type 2 diabetes. Science. 2004;306(5695):457-461.

18. Diano $\mathrm{S}$, et al. Peroxisome proliferation-associated control of reactive oxygen species sets melanocortin tone and feeding in diet-induced obesity. Nat Med. 2011;17(9):1121-1127.

19. McNay DE, Briancon N, Kokoeva MV, MaratosFlier E, Flier JS. Remodeling of the arcuate nucleus energy-balance circuit is inhibited in obese mice. J Clin Invest. 2012;122(1):142-152.

20. Balthasar N, et al. Divergence of melanocortin pathways in the control of food intake and energy expenditure. Cell. 2005;123(3):493-505.

\title{
Micro-editing mistake translates into a devastating brain tumor
}

\author{
Dan Dominissini, ${ }^{1,2}$ Ninette Amariglio, ${ }^{1}$ and Gideon Rechavi ${ }^{1,2}$
}

${ }^{1}$ Cancer Research Center, Chaim Sheba Medical Center, Tel Hashomer, Israel. ${ }^{2}$ Sackler School of Medicine, Tel Aviv University, Tel Aviv, Israel.

\begin{abstract}
RNA modifications are increasingly being recognized as critical players in cancer. While adenosine-to-inosine RNA editing is consistently deregulated in cancer, we are still unable to draw a straight line connecting transcriptspecific editing and carcinogenesis. The findings by Choudhury et al. in this issue of the JCI bridge this gap by mechanistically implicating underediting of miR-376a* in promoting glioma invasiveness through redirection of its mRNA targets. Moreover, RAP2A and AMFR convincingly emerge as key regulators of glioma migration and invasion affected by deregulated microRNA editing. Being inherently malleable, epigenetic mechanisms may provide feasible targets for therapeutic benefit.
\end{abstract}

Advances in the understanding of RNAbased regulatory pathways are revolutionizing biology and medicine. In this issue of the JCI, Choudhury et al. (1) describe the interplay of microRNAs (miRNAs), major players in cancer, with the adenosine-toinosine (A-to-I) RNA editing machinery.

Conflict of interest: The authors have declared that no conflict of interest exists.

Citation for this article: J Clin Invest. 2012;

122(11):3842-3845. doi:10.1172/JCI66178.

\section{MicroRNAs: small RNA molecules with a major role in cancer}

miRNAs constitute a new type of regulators of gene expression. Thousands of miRNAs have been identified in plant and animal genomes, including the human genome (reviewed in ref. 2). miRNA genes are usually transcribed into primary stem-loop structure precursors (pri-miRNAs) that are processed in the nucleus by Drosha-containing complexes into approximately 70-nt hairpin precursors (pre-miRNAs). Pre-miRNAs are exported to the cytoplasm and further trimmed by Dicer complexes, with loop removal resulting in approximately 21- to 23-nt-long mature miRNAs. One strand of the miRNA duplex is bound by an Argonaute protein to form a miRNA-induced silencing complex (miRISC) that regulates the expression of target mRNAs containing complementary sequences. Pairing between miRNAs and their cognate mRNA targets usually results in decreased protein expression, owing to mRNA degradation and translational repression. Most target mRNAs are regulated by miRNA binding to the $3^{\prime}$ untranslated regions (UTRs). The resulting miRNA/mRNA duplex includes a perfect pairing segment between a "seed," comprising nt 2-7 at the 5' end of the miRNA and the complementary target (2). This post-transcriptional regulatory mechanism is an integral part of most cellular pathways and gene networks. Aberrations of miRNAs biogenesis and function have 